\title{
A giant intrathoracic right subclavian artery aneurysm: a case report
}

\author{
Şebnem Albeyoğlu, Mustafa Aldağ, Ufuk Çiloğlu, Hakan Kutlu, Sabri Dağsalı
}

Received: April 21, 2016 Accepted: April 22, 2016 Published online: July 21, 2016

\begin{abstract}
Aneurysms of the subclavian artery are extremely rare in the clinical setting. Intrathoracic segmental aneurysms of the subclavian artery can occur secondary to atherosclerosis, cystic necrosis of the tunica media, trauma or infections. Open surgical repair is one of the main treatment choice. However, in case of intrathoracic segment involvement, it commonly necessitates a more invasive surgical approach with sternotomy or high lateral thoracotomy. Herein, we present a 40 -year-old male case with an expanding pulsatile mass throughout the right neck and dysphonia due to recurrent laryngeal nerve palsy. Computed tomography demonstrated an $8.5 \mathrm{~cm}$ in size proximal right subclavian artery saccular aneurysm with partial thrombosis. On angiography, the aneurysm was originating from about $1 \mathrm{~cm}$ after the origin of the right subclavian artery. As the aneurysm was very close to the brachiocephalic bifurcation, open surgical repair via a right supraclavicular incision with mini-sternotomy was performed. Pathological report of the aneurysmal sac was consistent with cystic medial degeneration.
\end{abstract}

Keywords: Subclavian artery; surgery; thoracic aneurysm.

Aneurysms of the subclavian artery, which are rarely seen in the clinical setting, represent less than $1 \%$ of all aneurysms. ${ }^{[1]}$ Subclavian artery aneurysms are classified based on their anatomical locations which typically reflects the etiology as intrathoracic and extra-thoracic aneurysms. ${ }^{[2]}$ Those in the extrathoracic site are more common and about three-quarters of aneurysms at this site are related to thoracic outlet syndrome or to previous injuries. ${ }^{[3]}$ Intrathoracic segmental aneurysms of the subclavian artery can occur secondary to atherosclerosis, cystic necrosis of the tunica media, trauma or infections. ${ }^{[2,5]}$ Open surgical repair is the one of the treatment option; however, in case of intrathoracic segment involvement, it necessitates a more invasive surgical approach with sternotomy or high lateral thoracotomy. Herein, we present a case with an expanding pulsatile mass throughout the right neck and dysphonia due to recurrent laryngeal nerve palsy.

\section{CASE REPORT}

A 40-year-old man was admitted with a threemonth history of pulsatile mass on his right neck and progressive dysphonia. His medical history did not reveal any chest trauma or pulmonary infection. On admission, his vital signs were normal and blood pressure was equal on both arms. His laboratory test results were nonspecific. His chest
X-ray demonstrated a superior mediastinal mass with a deviated trachea from the midline. The laryngoscopic findings confirmed a right vocal cord palsy and hoarseness, which were considered to be due to recurrent laryngeal nerve palsy. Computed tomography demonstrated an $8.5 \mathrm{~cm}$ in size proximal right subclavian artery saccular aneurysm with partial thrombosis (Figure 1). On angiography, the aneurysm was originating from about $1 \mathrm{~cm}$ after the origin of the right subclavian artery. Arcus aortography also revealed a saccular aneurysm beginning near the subclavian origin As the aneurysm was very close to the brachiocephalic bifurcation, open surgical repair via right supraclavicular incision with ministernotomy was planned. A written informed consent was obtained from the patient.

Repair of the aneurysm was performed through an incision with partial upper sternotomy (J-shaped incision to the upper third of the sternum) and the incision curved to right supraclavicular region with a $6 \mathrm{~cm}$ transverse skin incision (Figure 2).

Department of Cardiovascular Surgery, Siyami Ersek Thoracic and Cardiovascular Surgery Training and Research Hospital, İstanbul, Turkey

Corresponding author: Mustafa Aldağ, MD. Dr. Siyami Ersek Göğüs Kalp ve Damar Cerrahisi Eğitim ve Araştırma Hastanesi, Kalp ve Damar Cerrahisi Kliniği, 34668 Kadıköy, İstanbul, Turkey.

Tel: +90 532 - 3747170 e-mail: mustafa.aldag@saglik.gov.tr 


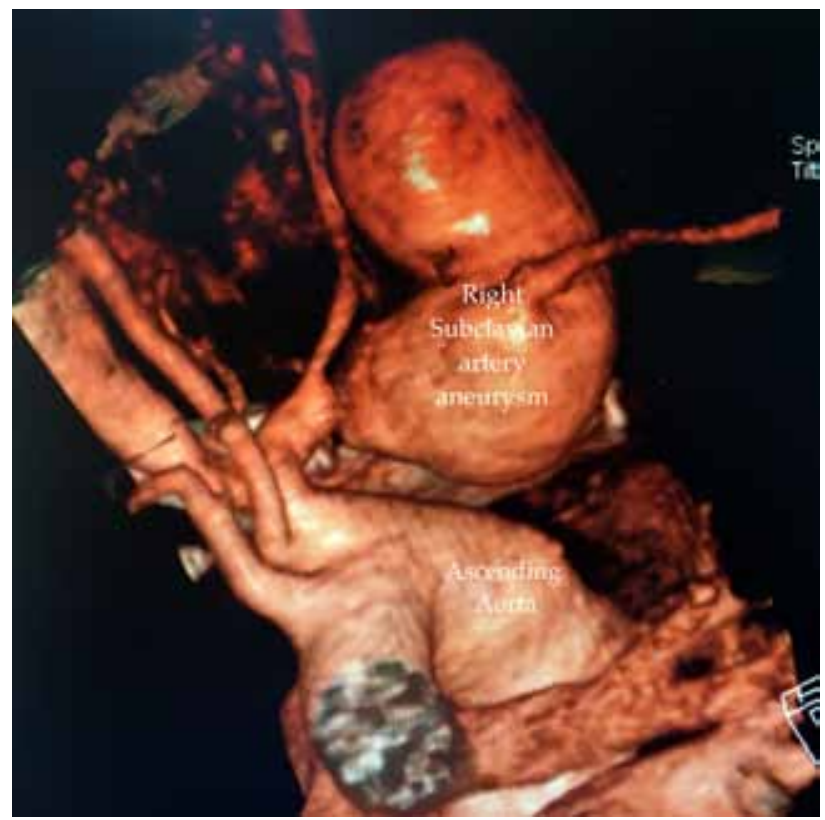

Figure 1. Computed tomography showing an $8.5 \mathrm{~cm}$ in size proximal right subclavian artery saccular aneurysm with partial thrombosis.

The ascending aorta and innominate artery were exposed, and the subclavian artery was dissected above the supraclavicular region. The aneurysmal sac was, then, dissected from the surrounding tissues. The right common carotid artery was tightly adhered to the aneurysmal sac and the right common carotid artery was carefully separated from the aneurysm.

Afferent and efferent loops of the aneurysm were dissected and secured. The aneurysm cavity was about $8 \times 8 \times 7 \mathrm{~cm}$ in size. After clamping, the aneurysm sac was opened. It was partially thrombosed and the origin of vertebral artery was able to be saved. Graft interpositioning between the proximal and distal parts of the subclavian artery was considered. As the vascular tissue was fragile, dissection was performed on the proximal part of the subclavian artery during the graft anastomosis. The dissection was progressed to the truncus of the brachiocephalic artery. We occluded the brachiocephalic artery at its origin on the arcus aorta.

The distal part of the subclavian artery was, then, interposed to the ascending aorta by an $8 \mathrm{~mm}$ in size expanded polytetrafluoroethylene (ePTFE) graft. Proximal anastomosis was done to the ascending aorta easily by a side biting clamp. Common carotid artery was separated from the innominate artery at its

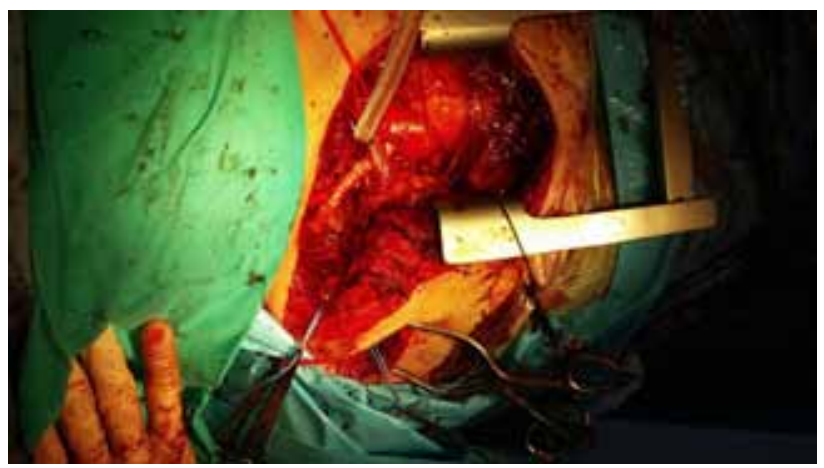

Figure 2. An intraoperative image showing mini-sternotomy and right supraclavicular incision.

origin. The distal end of the carotid artery was also anastomosed to the graft by end-to-side technique (Figure 3). Pathological report of the aneurysmal sac was consistent with cystic medial degeneration, a rare cause of an intrathoracic subclavian aneurysm (Figure 4). The microscopic findings were the basophilic ground substance in the media and disruption of the elastic lamina. Repeated digital subtraction angiography revealed a patent subclavian graft and carotid artery flow (Figure 5).

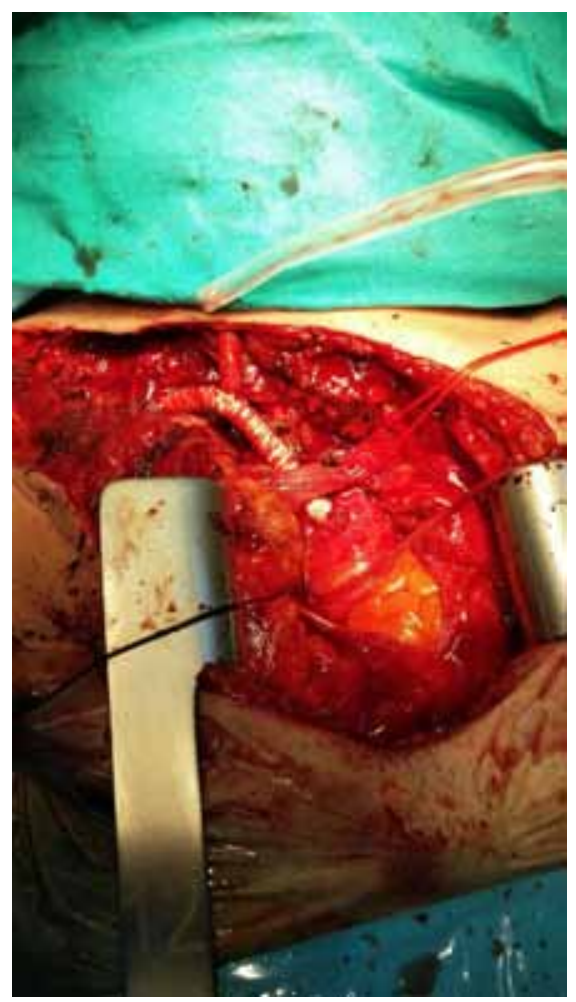

Figure 3. Subclavian artery graft and native carotid artery. 


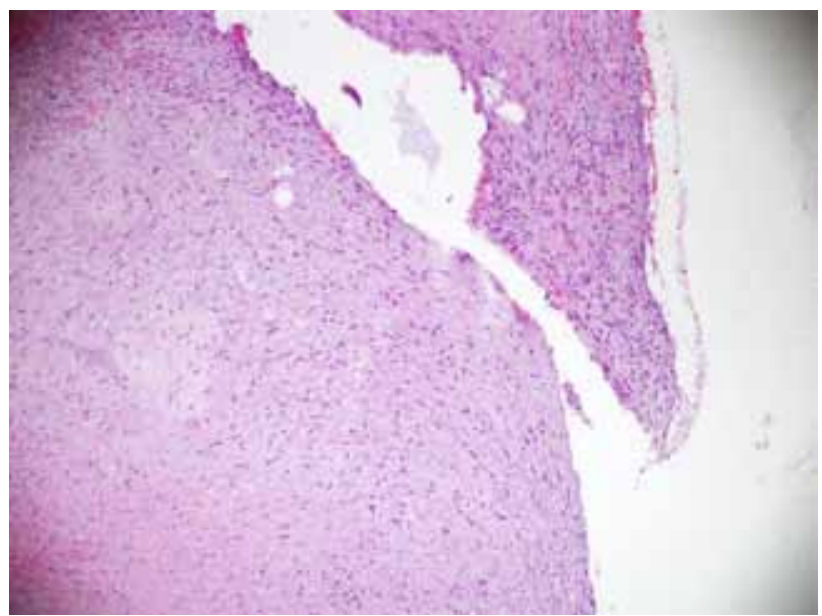

Figure 4. Pathology report of an aneurysmal sac consistent with cystic medial degeneration. Basophilic ground substance in the media and disruption of the elastic lamina.

\section{DISCUSSION}

Aneurysms of the subclavian artery accounts for about $\% 1$ of all peripheral arterial aneurysms. ${ }^{[1,2]}$ In a literature review, Hobson et al. ${ }^{[13]}$ identified 195 cases of aneurysms and found only $1 \%$ of all peripheral aneurysms originated from the subclavian artery. The aneurysms can be classified in terms of location on the subclavian artery, as extrathoracic and intrathoracic. ${ }^{[2]}$ While the aneurysms of extrathoracic subclavian artery are mostly related to thoracic outlet syndrome or to previous injuries (iatrogenic), intrathoracic aneurysms are mainly due to atherosclerosis or less often seen with cystic medial degeneration, Ehler Danlos syndrome, Marfan's syndrome, giant cell arteritis, Takayasu's arteritis, infection, or injuries. ${ }^{[5,7]}$ Herein, we discuss the surgical management of a young and non-atherosclerotic, vasculopathy in a case with a giant intrathoracic right subclavian artery aneurysm, as a rare pathological cause of intrathoracic subclavian aneurysms.

Although intrathoracic aneurysms of the subclavian artery are often asymptomatic, they may present with symptoms caused by compression or acute aneurysm expansion, such as upper chest or shoulder pain, tumor body can erode the apex of the lung may cause hemoptysis, compression to the recurrent laryngeal nerve resulting in hoarse voice; however, dyspnea caused by trachea compression is seldom reported..$^{[5]}$ Distal embolization to the upper limb and ischemia is an unusual complication.

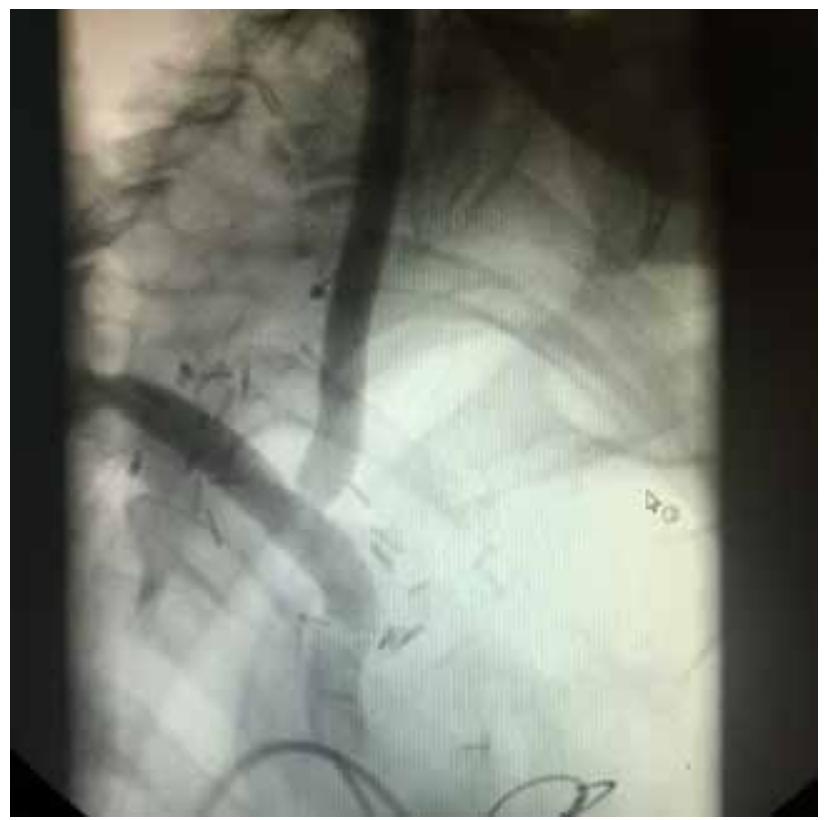

Figure 5. Postoperative digital subtraction angiography showing a patent subclavian graft and carotid artery flow.

Aneurysms of extrathoracic subclavian artery most commonly presents with a pulsatile mass in the superior fossa and is often tender. ${ }^{[6]}$ Brachial plexopathy and distal embolization are complications of the extrathoracic aneurysms. ${ }^{[3,4,6]}$

Elective surgical repair is the treatment of choice for most subclavian aneurysms, as they have an increased risk of rupture, embolization, thrombosis, and other complications ${ }^{[1,7,9]}$ Surgical approach for aneurysms of the left subclavian artery, a left-side lateral thoracotomy is used, for aneurysms on the right subclavian, are best approached by median sternotomy, improved control of the ascending aorta and the aneurysm's neck can be achieved with sternotomy with or without a supraclavicular incision and the sternoclavicular joint may or may not be disarticulated. ${ }^{[4,10]}$ The resection of the aneurysm is preferred to simple ligation, as continued growth and rupture of ligated aneurysms have been reported. ${ }^{[8,9]}$ Soylu et a1. ${ }^{[10]}$ reported a case presented with an intrathoracic giant subclavian artery aneurysm and the performed sternotomy and bypass with aortoasubclavian graft procedures successfuly.

We preferred combined mini-sternotomy and supraclavicular incision, as a safe and acceptable method for such young and non-atherosclerotic vasculopathy suspected patients. Fragile vasculature due to the connective tissue abnormality may cause 
some technical challenges during the operation, as in our case. Recently, as a less invasive alternative to surgical repair, endovascular stent grafting has become possible. However, it necessitates an acceptable proximal and distal neck, which serves as a proximal and distal landing zone for the stent graft. On the other hand, the upper extremity has an extensive collateral circulation which may lead to a potential risk for leakage during endoluminal treatment. ${ }^{[11,12]}$ Therefore, surgery still the standard treatment of choice in the literature in such cases. In our case, pathological report of the aneurysmal sac was reported as cystic medial degeneration and supported our suspicion for vasculopathy and open surgery decision.

In conclusion, surgical treatment should be preferred for young and non-atherosclerotic vasculopathy suspected patients. Aneurysmectomy in conjunction with graft interposition via mini-sternotomy and right supraclavicular incision is a safe and precautionary approach in such cases.

\section{Declaration of conflicting interests}

The authors declared no conflicts of interest with respect to the authorship and/or publication of this article.

\section{Funding}

The authors received no financial support for the research and/or authorship of this article.

\section{REFERENCES}

1. Dougherty MJ, Calligaro KD, Savarese RP, DeLaurentis DA. Atherosclerotic aneurysm of the intrathoracic subclavian artery: a case report and review of the literature. J Vasc Surg 1995;21:521-9.

2. Esteves FP, Ferreira AV, dos Santos VP, Novaes GS, Filho AR, Caffaro RA. Subclavian and axillary arterial aneurysms: two case reports. J Vasc Bras 2013;12: 329-34.

3. Zhan B, Zhang S, Shao Y. Operation for huge subclavian artery aneurysm: a case report. J Thorac Dis 2010;2:117-20.

4. Bond C, Muzaffar J, Adam D, Bishay E. Dartevelle approach for intra-thoracic subclavian artery aneurysm repair. Open Journal of Thoracic Surgery 2014;4:21-6.

5. Mechchat A, El Hammoumi MM, El Mesnaoui A, Lekehal B, Bensaid Y. Giant aneurysm of the right intra thoracic sub-clavian artery presenting as a dysphonia. Pan Afr Med J 2011;9:39.

6. Davidović LB, Marković DM, Pejkić SD, Kovacević NS, Colić MM, Dorić PM. Subclavian artery aneurysms. Asian J Surg 2003;26:7-11.

7. Bin HG, Kim MS, Kim SC, Keun JB, Lee JH, Kim SS. Intrathoracic aneurysm of the right subclavian artery presenting with hoarseness: a case report. J Korean Med Sci 2005;20:674-6.

8. Mukhopadhyay S, Roy S, Verma AK, Chowdhury G. Giant intrathoracic aneurysm of the right subclavian artery with tracheal compression following neck gland biopsy for tubercular lymphadenitis: Treated by partial cardiopulmonary bypass and grafting-A case report. IJTCVS 2009;25:75-7.

9. Lai SH, Ko PJ, Liao SL, Chung HT. A rare cause in an adolescent with chest pain: right intrathoracic subclavian artery aneurysm. J Pediatr Resp Dis 2014;10:40-3.

10. Soylu L, Bayazit M. Intrathoraric giant subclavian artery aneurysm: case report. Turkiye Klinikleri J Cardiovasc Sci 2013;25:191-4.

11. Andersen ND, Barfield ME, Hanna JM, Shah AA, Shortell $\mathrm{CK}, \mathrm{McCann} \mathrm{RL}$, et al. Intrathoracic subclavian artery aneurysm repair in the thoracic endovascular aortic repair era. J Vasc Surg 2013;57:915-25.

12. Harding GE, Kribs SW, Forbes TL. Hybrid open and endovascular therapy for a proximal subclavian artery aneurysm. Vascular 2008;16:236-8.

13. Hobson II RW, Israel MR, Lynch TG. Axilosubclavian arterial aneurysms. In: Bergan JJ, Yao JST, editors. Aneurisms - Diagnosis and Treatment. New York: Grune and Stratton; 1982. p. 435. 